\title{
Measuring Water Activity of Aviation Fuel Using a Polymer Optical Fiber Bragg Grating
}

\author{
Wei Zhang*a , David J. Webb ${ }^{\mathrm{a}}$, Mark Carpenter ${ }^{\mathrm{b}}$, Colleen Williams ${ }^{\mathrm{b}}$ \\ ${ }^{a}$ Aston Institute of Photonic Technologies, Aston University, Birmingham B4 7ET, UK; \\ b2CSFE Group, Defence Academy of the United Kingdom, Shrivenham SN6 8LA, UK
}

\begin{abstract}
Poly(methyl methacrylate) (PMMA) based polymer optical fiber Bragg gratings have been used for measuring water activity of aviation fuel. Jet A-1 samples with water content ranging from 100\% ERH (wet fuel) to 10 ppm (dried fuel), have been conditioned and calibrated for measurement. The PMMA based optical fiber grating exhibits consistent response and a good sensitivity of $59 \pm 3 \mathrm{pm} / \mathrm{ppm}$ (water content in mass). This water activity measurement allows PMMA based optical fiber gratings to detect very tiny amounts of water in fuels that have a low water saturation point, potentially giving early warning of unsafe operation of a fuel system.
\end{abstract}

Keywords: Polymer optical fiber, PMMA, fiber Bragg grating, water activity, equilibrium relative humidity

\section{INTRODUCTION}

Aviation fuel has the ability to hold a certain amount of dissolved water. The maximum amount of water that a given fuel can contain is referred to as its saturation point. This becomes critical when the water content nears the fuel's saturation point, creating a risk of actually exceeding the saturation point and forming free water - a destructive contaminant to almost all fuel applications. In an aviation fuel system (FS), water, in addition to not burning in an engine, will freeze at the low temperatures encountered in high altitude flights. The resulting ice may plug fuel filters and otherwise impede fuel flow. In addition to water already present in fuel, humid air in hazardous weather is also transformed to water in the fuel tanks leading to abnormal presence of water in the FS with increased risk for lightning damage (due to the water conductivity). Water in the fuel also may facilitate the corrosion of some metals and the growth of microorganisms. The solids formed by microbial growth are very effective at plugging fuel filters. Some microorganisms also generate acidic by-products that can accelerate metal corrosion. The best approach to microbial contamination is prevention and the most important preventive step is keeping the amount of free water in fuel storage tanks and aircraft fuel tanks as low as possible $[1,2]$. In order to have effective and efficient removal of free water it is important to monitor the water content in fuel.

A typical water-saturated fuel contains between 40 and $80 \mathrm{ppm}$ dissolved water at $21^{\circ} \mathrm{C}\left(70^{\circ} \mathrm{F}\right)$. If the temperature of the fuel increases, it can dissolve more water. Conversely, if the temperature of water-saturated fuel decreases, some of the water dissolved in the fuel will separate as free water. Compared to the amount of fuel the saturated water content is very small either in volume or in mass. This makes the measurement task difficult. There is currently a lack of a convenient, electrically passive system for water-in-fuel monitoring; instead the airlines rely on colorimetric spot tests or simply draining liquid from the bottom of fuel tanks. For all these reason, people have explored different ways to detect water in fuel, however all these approaches have problems, e.g. they may not be electrically passive or they may be sensitive to the refractive index of the fuel. The traditional unit of measurement for quantifying water content in fuel has been ppm (parts per million). It is an absolute moisture parameter that describes the volume or mass ratio of water to fuel. By actively measuring ppm levels of water in fuel, the absolute amount of water can be determined. However, a ppm measurement has one major limitation - it does not account for any variation in a fuel's saturation point. In other words, in a dynamic fuel system with a fluctuating saturation point, a ppm measurement would provide no indication of how close the moisture level is to the fuel's saturation point. Water activity is the amount of water in a substance relative to the total amount of water it can hold. Regardless of the saturation point of the fluid, a water activity reading will always provide a true indication of risk of free water formation. In this work we propose a technique using a PMMA based optical fiber grating for measuring the water activity of aviation fuel.

*w.zhang@aston.ac.uk

23rd International Conference on Optical Fibre Sensors, edited by José Miguel López-Higuera,

Julian Jones, Manuel López-Amo, José Luis Santos, Proc. of SPIE Vol. 9157, 91574V

(C) 2014 SPIE · CCC code: 0277-786X/14/\$18 · doi: 10.1117/12.2059273 


\section{PRINCIPLE OF MEASUREMENT}

Water activity is defined as [3]

$$
a_{W}=P_{S} / P_{O}
$$

where $P_{S}$ is the vapor pressure of water above a sample and $P_{O}$ that of pure water at the same temperature. Water activity values represent a scale that ranges from 0 (bone dry) to 1.0 (pure water). In a fuel system $a_{W}=0$ means dry fuel and $a_{W}=1$ water saturated fuel. From its definition water activity is equal to equilibrium relative humidity (ERH).

The maximum amount of water that a given fuel can contain in solution is referred to as its saturation point. A fuel's saturation point is a function of many different factors such as the composition as well as the type of additives present. The equation below provides the estimation for water solubility in fuel (percentage by mass) [1]:

$$
S=S_{0} H\left(\frac{P}{P_{0}}\right)\left(\frac{T}{T_{0}}\right)^{m}
$$

where $S_{0}$ is the maximum solubility of water in the fuel at the reference condition of $H=1.0$ (100\% relative humidity the saturation point), $H$ is the humidity of air above the fuel, $P$ the air pressure above the fuel, $T$ the temperature of the air and fuel, $m$ a constant depending on the fuel, $P_{0}=0.1 \mathrm{MPa}$ and $T_{0}=293 \mathrm{~K}$. At the specified temperature and air pressure the water solubility is proportional to the environmental humidity.

On the other hand PMMA can absorb a certain amount of water. Water absorption in PMMA as derived from the multimolecular theory of absorption, can be expressed as [4],

$$
s=\frac{s_{1} c x}{1-x}\left[\frac{1-(n+1) x^{n}+n x^{n+1}}{1+(c-1) x-c x^{n+1}}\right]
$$

where $s$ is the weight of absorbate per gram of adsorbent, $\mathrm{s}_{1}$ the weight of absorbate per gram of absorbent when each absorption site is covered by one mole of absorbent, $c$ and $n$ are constants; $x=P_{S} / P_{O}$ where $P_{S}$ is the equilibrium absorption pressure in the absorbate and $P_{O}$ the saturation pressure over a free liquid surface of the absorbate. Here the absorbate is water and the absorbent PMMA. Therefore the ratio of these two parameters represents the equilibrium relative humidity. For the polymethyl methacrylate (Perspex), $w_{1}$ is $6.25, c=1, n=5$. According to (2) water absorption in PMMA is a function of the equilibrium relative humidity and can be calculated.

Water absorption introduces changes in both the volume and refractive index of PMMA. For a PMMA based polymer optical fiber Bragg grating (POFBG), its Bragg wavelength depends on the effective core refractive index $n_{\text {eff }}$, and the grating pitch $\Lambda$, both of which can be modulated by the water content in PMMA optical fiber. At a specified temperature the wavelength change of POFBG against relative humidity can be expressed as

$$
\lambda_{B}=2 n_{e f f}\left(a_{W}\right) \Lambda\left(a_{W}\right)
$$

This indicates that the POFBG can detect the change of water activity when placed in aviation fuel.

\section{EXPERIMENTS AND RESULTS}

An experimental arrangement was set up to control the dissolved water content in fuel and investigate the performance of a POFBG for water detection in fuel, as shown in Fig. 1. POFBG sensors were fabricated by attaching a $10 \mathrm{~cm}$ length of POF to a single mode silica fiber down-lead using UV curable glue (Norland 78), as described in [5]. For testing, the POFBG sensors were placed inside an environmental chamber (Sanyo Gallenkamp). They were illuminated via a fiber optic circulator with light from a broadband light source (Thorlab ASE730) and observed in reflection using an IBSEN IMON 400 wavelength interrogation system.

As shown by (2), the amount of dissolved water depends on the relative humidity of the air above the fuel. It assumes that the fuel is in equilibrium with free water or moist air. Fuel close to a fuel-water or fuel-air interface will reach water equilibrium in a matter of minutes. Therefore the water content in a fuel sample can be varied by exposing fuel to humid air. Different amounts of water in fuel can be conditioned by changing the surrounding relative humidity. 
Jet-A1 aviation fuel was held in a beaker placed in the environmental chamber to be in contact with air that can be set to a chosen temperature and humidity. The POFBG was inserted into the fuel and monitored under different relative humidities.

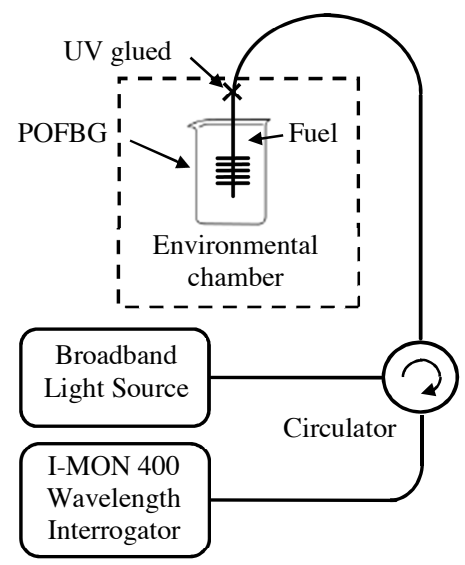

Fig. 1. Experimental arrangement for using a POFBG to measure the water activity of aviation fuel.

First the temperature of the environmental chamber was set at $24.5^{\circ} \mathrm{C}$ and the relative humidity was step changed from $45 \%$ through $60 \%$ and $75 \%$, to $90 \%$. Since it takes time for fuel to reach water equilibrium the chamber was kept at each humidity value for a long time (up to 4 hours at each humidity setting in the experiment). The measured wavelength response of the POFBG in fuel is shown in Fig. 2a, in which the humidity value measured by the chamber's built-in sensor is plotted as well. From it one can see that for each humidity setting it takes nearly 2 hours for the POFBG response to reach a stable value, though this long response time is attributed to the time needed to achieve water equilibrium in the fuel, rather than the response time of the POFBG itself which is typically much faster [5]. This equilibrium time closely depends on the volume and the geometry of the container of the fuel. If the volume of fuel is large and the area of the interface between fuel and air is limited it will take a lot longer to reach water equilibrium. In the bottoms of some fuel tanks it never reaches equilibrium.

In order to accelerate the water equilibration process in the fuel, a magnetic stirrer was used to stir the fuel in the beaker. The wavelength response of POFBG in fuel was monitored while the relative humidity of the chamber was set to different values. Fig. $2 \mathrm{~b}$ shows a typical response of the POFBG sensor recorded while the humidity was changed in a step of $10 \%$ from $40 \%$ to $90 \%$ and the chamber temperature was kept at $24.5^{\circ} \mathrm{C}$. Clearly the response of the system is much faster.

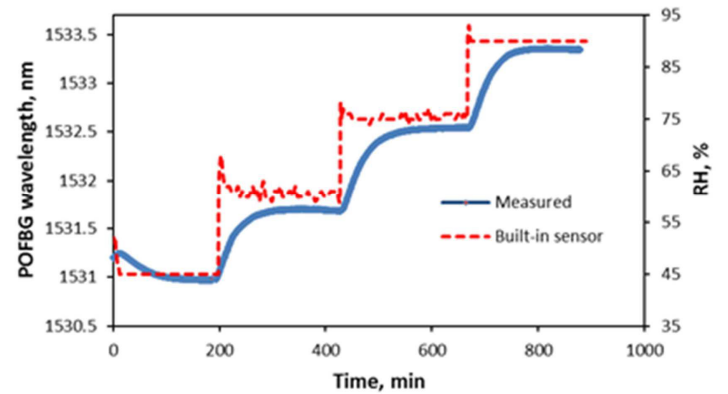

(a)

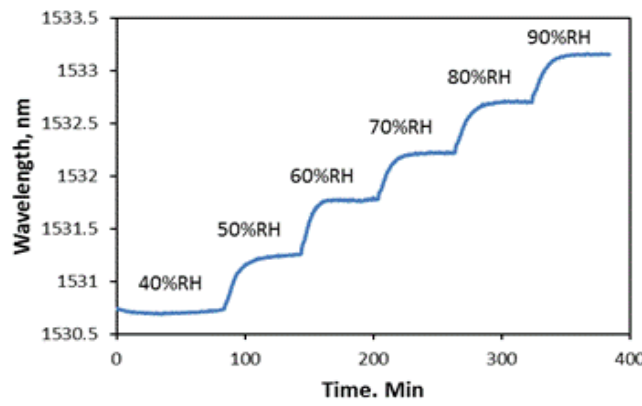

(b)

Fig. 2 POFBG wavelength response against varying ERH. (a) fuel not stirred, (b) fuel stirred

Three samples of fuel were further used to test the POFBG sensor performance. The fuel was dispensed into three small glass vials, each of which contained about $20 \mathrm{ml}$ of Jet A-1. Sample 1 was dried, as far as possible, by placing the vial in a desiccator which contained a bed of silica gel. Sample 2 was left open and exposed to ambient air. Sample 3 was exposed to a $100 \% \mathrm{RH}$ atmosphere by placing the vial in a desiccator that contained a small amount of distilled water. On conclusion of the conditioning period, the water content of each sample of fuel was measured using a Karl Fischer 
(KF) coulometer. The following measurements were recorded: $10 \mathrm{ppm}$ dissolved water in sample 1 (by mass); 39 ppm dissolved water in sample 2; $68 \mathrm{ppm}$ dissolved water in sample 3. These samples were actually processed in the same way as had previously been done in the environmental chamber, but with known water content measured by using the Karl Fischer (KF) coulometer. The recorded response in Fig. 3 was measured using a POFBG sensor. Again it shows a fast response time.

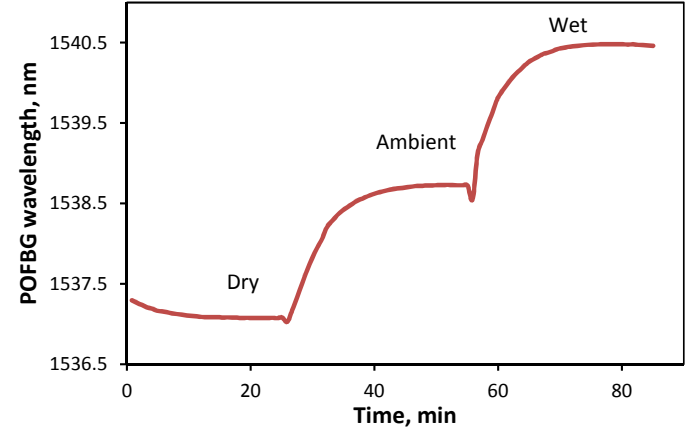

Fig. 3 POFBG response of 3 calibrated fuel samples

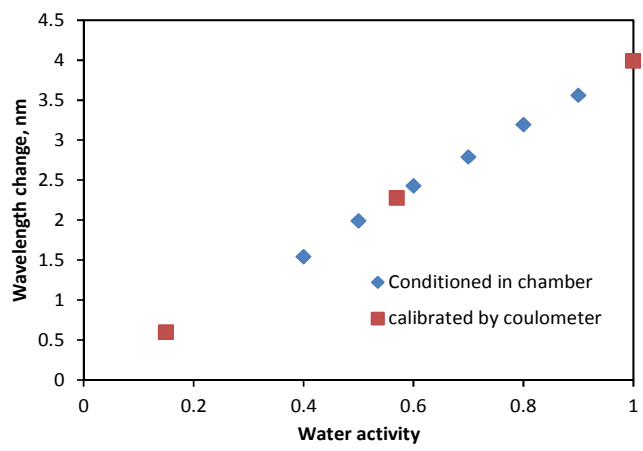

Fig. 4 POFBG wavelength change vs. water activity of fuel

The wavelength change of the POFBG is plotted against the water activity of fuel in Fig. 4. The results for the fuel conditioned in the environmental chamber and the calibrated fuel samples (conditioned in the desiccator) show very good agreement and consistent sensitivity. This change can be linked to the water content in the fuel by Equation (2) as far as the constants in (2) are known. These constants depend on the particular type of fuel. For the fuel used in this work (Air BP Jet A-1) the maximum water solubility at different temperature has been measured [1]. Based on the measured results the sensitivity of water content detection in fuel using a POFBG can be estimated as $59 \pm 3 \mathrm{pm} / \mathrm{ppm}$ for a grating at $1535 \mathrm{~nm}$.

\section{CONCLUSION}

In this work we have investigated POFBGs for detecting the water content in aviation fuel. The POFBG sensor is capable of directly measure the water activity of fuel. This particular feature of measurement allows a PMMA based optical fiber grating to detect very tiny amounts of water in fuels that have low water saturation point and potentially give early warning of the unsafe operation of a fuel system. The results have shown consistent response and good sensitivity. According to the results the sensitivity of water content detection in fuel using a POFBG can be estimated as $59 \pm 3 \mathrm{pm} / \mathrm{ppm}$ for a grating at $1535 \mathrm{~nm}$. The wavelength resolution for the current I-MON wavelength interrogation system is about $1 \mathrm{pm}$. This means that the minimum detectable water content in fuel should be better than $0.1 \mathrm{ppm}$ at constant temperature. This is much better performance than any available water content detection measure.

\section{ACKNOWLEDGEMENT}

The research leading to these results has received funding from the European Union Seventh Framework Programme (FP7/2007-2013) under grant agreement no. 314032.

\section{REFERENCES}

[1] [Handbook of aviation fuel properties], CRC Inc. (1983)

[2] Aviation fuels technical review (www.cgabusinessdesk.com/document/aviation_tech_review.pdf) (2006).

[3] Barbosa-Cánovas, G. V., Fontana, Jr., A. J., Schmidt, S. J., Labuza, T. P., [Water Activity in FoodsFundamentals and Applications], Blackwell Publishing, (2007).

[4] Turner, D. T., "Polymethyl methacrylate plus water: sorption kinetics and volumetric changes," Polymer, 23, 197-202 (1982).

[5] Zhang, W., Webb, D. J., and Peng, G. D., "Investigation into time response of polymer fiber Bragg grating based humidity sensors,” J. Lightwave Technol., 30, 1090-1096 (2012). 